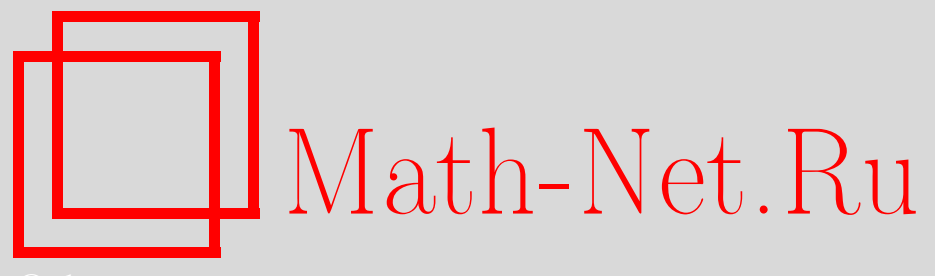

И. А. Чеплюкова, Об одной характеристике случайного отображения с известным числом циклов, Дискрет. матем., 2006, том 18, выпуск 3, 43-60

DOI: https://doi.org/10.4213/dm58

Использование Общероссийского математического портала Math-Net.Ru подразумевает, что вы прочитали и согласны с пользовательским соглашением http://www.mathnet.ru/rus/agreement

Параметры загрузки:

IP: 35.173 .219 .12

26 апреля 2023 г., 09:01:33

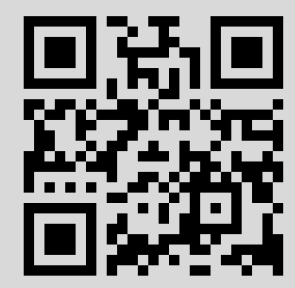




\title{
Об одной характеристике случайного отображения с известным числом циклов
}

() 2006 г. И. А. Чеплюкова

\begin{abstract}
Рассматривается случайная величина, равная числу деревьев заданного объема в графе случайного однозначного отображения множества $n$ элементов в себя, содержащем $m$ компонент связности. Получены предельные теоремы описывающие распределение этой характеристики в случае, когда $n \rightarrow \infty, m / \ln n \rightarrow \infty, m / \ln n=O(\ln n)$.

Работа выполнена при поддержке программой Президента Российской Федерации государственной поддержки ведущих научных школ Российской Федерации, грант НШ 1758.2003.1, и гранта 2005 г. Фонда содействия отечественной науке.
\end{abstract}

Рассмотрим однозначное отображение множества из $n$ элементов в себя, граф которых содержит $m$ компонент связности. На множестве $\Sigma_{n, m}$ всех таких отображений введем равномерное распределение вероятностей. Тогда числовые характеристики этих отображений являются случайными величинами. Если в графе отображения из $\Sigma_{n, m}$ удалить ребра, соединяющие циклические вершины, то оставшаяся часть графа образует лес. Поэтому естественно использовать для получения результатов о случайных отображениях известные результаты о случайных лесах. Эта идея была впервые использована Ю. Л. Павловым в $[1,2]$. Здесь мы будем рассматривать множество лесов $\mathscr{F}$, состоящих из $N$ корневых деревьев, корни которых имеют номера $1,2, \ldots, N$, и из $n-N$ некорневых вершин, имеющих номера $1, \ldots, n-N$, при условии, что распределение вероятностей, заданное на $\mathscr{F}$, является равномерным. В [3] показано, что такому случайному лесу соответствует ветвящийся процесс с пуассоновским распределением числа прямых потомков каждой частицы. Обозначим $x$ параметр этого распределения и положим $x=(n-N) / n \exp \{-(n-N) / n\}$.

Через $\lambda^{(n, m)}$ обозначим число циклических вершин графа случайного отображения объема $n$ с $m$ циклами. Если распределение $\lambda^{(n, m)}$ известно, то, усредняя по нему распределения различных характеристик случайного леса, можно получить распределения соответствующих характеристик случайного отображения (см. [3]). Через $\lambda_{r}^{(n, m)}$ обозначим случайную величину, равную числу деревьев, содержащих $r$ некорневых вершин в случайном отображении с $m$ циклами. В случае ограниченного отношения $m / \ln n$ предельное поведение случайной величины $\lambda_{r}^{(n, m)}$ было получено в [3]. В настоящей работе мы дополним эти результаты, исследовав поведение $\lambda_{r}^{(n, m)}$ при $n \rightarrow \infty$ так, что $m / \ln n \rightarrow \infty, m / \ln n=O(\ln n)$. 
Введем обозначения

$$
\begin{aligned}
& \alpha=\frac{\sqrt{n}}{m}, \quad \quad \beta=\frac{m}{\ln \alpha}, \quad \mu=\frac{\beta}{2}\left(1-\frac{\ln \ln \alpha}{\ln \alpha}\right), \\
& p_{r}=\frac{(r+1)^{r}}{(r+1) !} \exp \{-(r+1)\}, \quad r=0,1, \ldots, \\
& b=p_{r} \sqrt{n /(2 \mu)}, \quad \gamma=\sqrt{(b+2) / b}, \quad \theta=p_{r} \sqrt{2 \mu n} .
\end{aligned}
$$

Кроме того, будем писать $f(n) \asymp g(n)$ (см. [4]), если неравенства $|f(n)| \leqslant C_{1}|g(n)|$ и $|g(n)| \leqslant C_{2}|f(n)|$ выполняются для всех достаточно больших $n$ (здесь и далее $C_{1}, C_{2}, \ldots$ означают некоторые положительные постоянные). Выражение $f(n) \succ g(n)$ означает, что $\lim _{n \rightarrow \infty} g(n) / f(n)=0$.

Справедливы следующие утверждения.

Теорема 1. Пусть $n \rightarrow \infty$ так, что $m / \ln n \rightarrow \infty, m / \ln n=O(\ln n), r=o\left((\mu n)^{1 / 3}\right)$. Тогда

$$
\gamma p_{r} \sqrt{n} \mathbf{P}\left\{\lambda_{r}^{(n, m)}=k\right\}=\frac{1}{\sqrt{\pi}} e^{-z^{2} / 2}+o(1)
$$

равномерно относительно чельх положительных $k$ таких, что $z=\left(k /\left(p_{r} \sqrt{n}\right)-\sqrt{2 \mu}\right) / \gamma$ лежст в любом фиксированном конечном интервале.

Теорема 2. Если $n \rightarrow \infty$ так, что $m / \ln n \rightarrow \infty, m / \ln n=O(\ln n), r \asymp(\mu n)^{1 / 3}$, то для любого $k=0,1,2, \ldots$

$$
\mathbf{P}\left\{\lambda_{r}^{(n, m)}=k\right\}=\frac{1}{k !} \theta^{k} e^{-\theta}(1+o(1)) .
$$

Теорема 3. Пусть $n \rightarrow \infty$ так, что $m / \ln n \rightarrow \infty, m / \ln n=O(\ln n), r \succ(\mu n)^{1 / 3}$. Тогда

$$
\mathbf{P}\left\{\lambda_{r}^{(n, m)}=0\right\}=1+o(1) .
$$

Приведем несколько теорем, с помощью которых докажем теоремы 1-3. В [5] доказаны следующие утверждения (теорема 4 и 5 ).

Теорема 4. Если $n \rightarrow \infty, m / \ln n \rightarrow \infty, m / \ln n=O(\ln n), z=N / \sqrt{n}, m o$

$$
\beta^{\mu} e^{-\beta / 2} \sqrt{\pi n} \mathbf{P}\left\{\lambda^{(n, m)}=N\right\}=z^{2 \mu} e^{-z^{2} / 2}(1+o(1))
$$

равномерно относительно чельх $N$, для которых $z-\sqrt{2 \mu}$ лежит в любом фиксированном конечном интервале, где $\alpha, \beta$, $\mu$ определены в (1).

Пусть $\xi^{(n)}$ обозначает число циклов отображения множества объема $n$ в себя.

Теорема 5. Пусть $n \rightarrow \infty, \beta \rightarrow \infty, m / \ln n=O(\ln n)$. Тогда

$$
\frac{(m-1) ! \sqrt{2 \beta n}}{\left(-\ln \left(1-x_{1}\right)\right)^{m-1} e^{\beta / 2} \beta^{\mu}} \mathbf{P}\left\{\xi^{(n)}=m\right\}=e^{-\gamma_{1}}(1+o(1)),
$$

2de

$$
x_{1}=1-\frac{1}{\alpha \ln \alpha}, \quad \gamma_{1}=\frac{\beta}{8 \ln \alpha} \ln ^{2}(2 \mu),
$$

$a \alpha, \beta, \mu$ определены в (1). 
Обозначим $\lambda_{r}(\mathscr{F})$ число деревьев леса из $\mathscr{F}$, содержащих $r$ некорневых вершин. Пусть

$$
\begin{aligned}
p_{r}(x) & =\frac{(r+1)^{r}}{(r+1) !}\left(\frac{n-N}{n} e^{-(n-N) / n}\right)^{r} e^{-(n-N) / n}, \\
\sigma_{r r}^{2} & =p_{r}(x)\left(1-p_{r}(x)-\left(\frac{n-N}{N}-r\right)^{2} p_{r}(x) N^{3} /\left((n-N) n^{2}\right)\right) .
\end{aligned}
$$

В [3] доказана следующая теорема для случайной величины $\lambda_{r}(\mathscr{F})$.

Теорема 6. Пусть $N, n, r \rightarrow \infty$, тогда для иельх неотрицательных $k$

$$
\mathbf{P}\left\{\lambda_{r}(\mathscr{F})=k\right\}=(k !)^{-1}\left(N p_{r}(x)\right)^{k} \exp \left\{-N p_{r}(x)\right\}(1+o(1))
$$

равномерно относительно $\left(k-N p_{r}(x)\right) / \sqrt{N p_{r}(x)}$ в любом конечном интервале.

Перейдем к доказательству теорем 1-3.

Пусть выполнены условия теоремы 1. Доказательство этого утверждения разделим на несколько случаев:

(1) $r$ фиксировано;

(2) $r=o\left(n^{1 / 3} / \mu^{6 / 5}\right)$;

(3) $r \asymp\left(n^{1 / 3} / \mu^{6 / 5}\right)$;

(4) $r \succ n^{1 / 3} / \mu^{6 / 5}, r=o\left((n / \mu)^{1 / 3}\right)$;

(5) $r \asymp(n / \mu)^{1 / 3}$;

(6) $r \succ(n / \mu)^{1 / 3}$.

Рассмотрим первый случай. В [3] было показано, что

$$
\mathbf{P}\left\{\lambda_{r}^{(n, m)}=k\right\}=\sum_{N=k}^{n-k r} \mathbf{P}\left\{\lambda^{(n, m)}=N\right\} \mathbf{P}\left\{\lambda_{r}(F)=k\right\} .
$$

Из соотношений (1) видно, что $\gamma=1+o(1)$ при фиксированном $r$. Тогда

$$
\begin{aligned}
\gamma p_{n} \sqrt{n} \mathbf{P}\left\{\lambda_{r}^{(n, m)}=k\right\} & =p_{r} \sqrt{n} \sum_{N=k}^{n-k r} \mathbf{P}\left\{\lambda^{(n, m)}=N\right\} \mathbf{P}\left\{\lambda_{r}(\mathscr{F})=k\right\}(1+o(1)) \\
& =\sum_{i=1}^{4} S_{i}(1+o(1))
\end{aligned}
$$

где

$$
\begin{aligned}
& S_{i}= p_{r} \sqrt{n} \sum_{N_{i}} \mathbf{P}\left\{\lambda^{(n, m)}=N\right\} \mathbf{P}\left\{\lambda_{r}(\mathscr{F})=k\right\}, \quad i=1, \ldots, 4, \\
& N_{1}=\left\{N: k \leqslant N<(\sqrt{2 \mu}+z) \sqrt{n}-(2 \mu)^{1 / 4} n^{7 / 24} p_{r}^{-5 / 12}\right\}, \\
& N_{2}=\left\{N:(\sqrt{2 \mu}+z) \sqrt{n}-(2 \mu)^{1 / 4} n^{7 / 24} p_{r}^{-5 / 12} \leqslant N \leqslant(\sqrt{2 \mu}+z) \sqrt{n}\right. \\
&\left.\quad+(2 \mu)^{1 / 4} n^{7 / 24} p_{r}^{-5 / 12}\right\}, \\
& N_{3}=\left\{N:(\sqrt{2 \mu}+z) \sqrt{n}+(2 \mu)^{1 / 4} n^{7 / 24} p_{r}^{-5 / 12}<N \leqslant n^{2 / 3}\right\}, \\
& N_{4}=\left\{N: n^{2 / 3}<N \leqslant n-k r\right\} .
\end{aligned}
$$


Основной вклад в сумму, стоящую в правой части (4), дает слагаемое $S_{2}$. Из теоремы 4 следует, что

$$
S_{2}=p_{r} e^{-z^{2}} \frac{1}{\sqrt{\pi}} \sum_{N_{2}} \mathbf{P}\left\{\lambda_{r}(\mathscr{F})=k\right\}(1+o(1))
$$

Известно (см. [1]), что $p_{r}(x)=p_{r}(1+o(1))$ и $\sigma_{r r}^{2}=p_{r}\left(1-p_{r}\right)(1+o(1))$ при фиксированном $r$ в области $n / N \rightarrow \infty$, где $p_{r}$ определена в (1). Тогда из теоремы 3.1 .2 [3] и соотношения (6) находим, что

$$
S_{2}=p_{r} e^{-z^{2}} \frac{1}{\sqrt{\pi}} \sum_{N_{2}} \frac{1+o(1)}{\sqrt{2 \pi N p_{r}\left(1-p_{r}\right)}} \exp \left\{-\frac{\left(k-N p_{r}\right)^{2}}{N p_{r}\left(1-p_{r}\right)}\right\} .
$$

Полагая

$$
N=(z+\sqrt{2 \pi}) \sqrt{n}+y(2 \mu n)^{1 / 4} \sqrt{\left(1-p_{r}\right) / p_{r}},
$$

из (7) нетрудно получить, что

$$
S_{2}=(1+o(1)) \frac{e^{-z^{2}}}{\pi \sqrt{2}} \sum_{y} \sqrt{\frac{p_{r}}{1-p_{r}}} \frac{1}{(2 \mu n)^{1 / 4}} e^{-y^{2}},
$$

где суммирование по $y$ осуществляется в области $|y| \leqslant n^{1 / 24} p_{r}^{1 / 12} / \sqrt{1-p_{r}}$ с шагом $(2 \mu n)^{-1 / 4} \sqrt{p_{r} /\left(1-p_{r}\right)}$. Заменяя суммирование интегрированием, находим, что

$$
S_{2}=(1+o(1)) \frac{e^{-z^{2}}}{\pi \sqrt{2}} \int_{-\infty}^{\infty} e^{-y^{2} / 2} d y=\frac{e^{z^{2}}}{\sqrt{\pi}}(1+o(1)) .
$$

Рассмотрим сумму $S_{3}$. Обозначим через $\lambda^{(n)}$ число циклических точек отображения множества объема $n$ в себя (без ограничения на число циклов), а через $\varkappa_{N}-$ случайную величину, равную числу циклов в случайной подстановке степени $N$. В [3] показано, что

$$
\mathbf{P}\left\{\lambda^{(n, m)}=N\right\}=\frac{\mathbf{P}\left\{\lambda^{(n)}=N\right\} \mathbf{P}\left\{\varkappa_{N}=m\right\}}{\mathbf{P}\left\{\xi^{(n)}=m\right\}} .
$$

Тогда

$$
S_{3}=\operatorname{pr} \sqrt{n} \sum_{N_{3}} \frac{\mathbf{P}\left\{\lambda^{(n)}=N\right\} \mathbf{P}\left\{\varkappa_{N}=m\right\}}{\mathbf{P}\left\{\xi^{(n)}=m\right\}} \mathbf{P}\left\{\lambda_{r}(F)=k\right\}(1+o(1)) .
$$

Из теоремы 4.2.6 из [6] следует, что

$$
\mathbf{P}\left\{\varkappa_{N}=m\right\} \leqslant C_{3}\left(\ln \left(\frac{N}{m} \ln \frac{N}{m}\right)\right)^{m-1} \sqrt{\ln \frac{N}{m}} \exp \left\{\frac{m}{\ln (N / m)}\right\}((m-1) ! N \sqrt{n})^{-1},
$$

а из теоремы 5 вытекает, что

$$
\mathbf{P}\left\{\xi^{(n)}=m\right\} \geqslant C_{4} \frac{e^{\beta / 2-\gamma_{1}} \beta^{\mu}(\ln (\alpha \ln \alpha))^{m-1}}{\sqrt{n \beta}(m-1) !},
$$


где $\beta$ и $\mu$ определены в (1), а $\gamma_{1}$ определена в формулировке теоремы 5.

Обозначим через $\xi_{1}, \ldots, \xi_{N}$ независимые одинаково распределенные случайные величины, имеющие распределение вероятностей $\mathbf{P}\left\{\xi_{1}=j\right\}=p_{j}, j=0,1, \ldots$, где величины $p_{j}$ определены в (1). Через $\xi_{i}^{(r)}, i=1, \ldots, N, r=0,1, \ldots$, обозначим независимые случайные величины, распределения которых задаются вероятностями $\mathbf{P}\left\{\xi_{i}^{(r)}=j\right\}=\mathbf{P}\left\{\xi_{i}=j \mid \xi_{i} \neq r\right\}$. Обозначим также $v=\xi_{1}+\ldots+\xi_{N}$, $\zeta_{s}^{(r)}=\xi_{1}^{(r)}+\ldots+\xi_{s}^{(r)}$. Согласно лемме 3.1 .1 из [3],

$$
\mathbf{P}\left\{\lambda_{r}(F)=k\right\}=\left(\begin{array}{l}
N \\
k
\end{array}\right) p_{r}^{k}(x)\left(1-p_{r}(x)\right)^{N-k} \frac{\mathbf{P}\left\{\zeta_{N-k}^{(r)}=n-k(r+1)\right\}}{\mathbf{P}\{v=n\}} .
$$

Из (1) и (2) нетрудно видеть, что при $N / n \rightarrow 0$

$$
p_{r}(x)=p_{r} \exp \left\{\frac{N}{n}+O\left(\frac{r N^{2}}{n^{2}}\right)\right\}=p_{r}\left(1+\frac{N}{n}+O\left(\frac{r N^{2}}{n^{2}}\right)\right) .
$$

Из леммы 5 из [1] находим, что

$$
\mathbf{P}\{v=n\}=\frac{N}{\sqrt{2 \pi(n-N) n}}(1+o(1)) .
$$

Отсюда получаем, что

$$
\frac{\mathbf{P}\left\{\zeta_{N-k}^{(r)}=n-k(r+1)\right\}}{\mathbf{P}\{v=n\}} \leqslant C_{5} \frac{n^{3 / 2}}{N} .
$$

Кроме того, известно (см., например,[7]), что

$$
\mathbf{P}\left\{\lambda^{(n)}=N\right\} \leqslant N / n .
$$

Из соотношений (9), (11)-(16) получаем, что

$$
S_{3} \leqslant C_{6} n p_{r} e^{m+\beta-\gamma_{1}} \beta^{-\mu} \sum_{N_{3}}\left(\begin{array}{l}
N \\
k
\end{array}\right) p_{r}^{k}(x)\left(1-p_{r}(x)\right)^{N-k} .
$$

Нетрудно показать, что выражение $\left(\begin{array}{c}N \\ k\end{array}\right) p_{r}^{k}(x)\left(1-p_{r}(x)\right)^{N-k}$ монотонно убывает при $N>k / p_{r}(x)$. Обозначим

$$
N_{0}=(\sqrt{2 \mu}+z) \sqrt{n}+(2 \mu)^{1 / 4} n^{7 / 24} p_{r}^{-5 / 12} .
$$

Поскольку $N_{0}<N$ при $N \in N_{3}$ и $N_{0}>k / p_{r}(x)$, используя нормальное приближение для биномиального распределения, находим, что

$$
\begin{aligned}
S_{3} & \leqslant C_{7} n p_{r} e^{m+\beta / 2+\gamma_{1}} \beta^{-\mu} \sum_{N_{3}} \frac{1}{\sqrt{2 \pi N_{0} p_{r}(x)\left(1-p_{r}(x)\right)}} \exp \left\{-\frac{\left(k-N_{0} p_{r}(x)\right)^{2}}{2 N_{0} p_{r}(x)\left(1-p_{r}(x)\right)}\right\} \\
& \leqslant C_{7} n^{5 / 3} p_{r} e^{m+\beta / 2+\gamma_{1}} \beta^{-\mu} \frac{1}{\left(\sqrt{2 \mu n} p_{r}\right)^{1 / 2}} \exp \left\{-C_{8} n^{1 / 12} p_{r}^{1 / 6}\right\} \\
& =C_{7} \exp \left\{-C_{8} n^{1 / 12} p_{r}^{1 / 6}(1+o(1))\right\} \rightarrow 0 .
\end{aligned}
$$


Для оценки суммы $S_{4}$ используем неравенство, полученное при доказательстве теоремы 5 (см.[5]):

$$
\frac{\sum_{N_{4}} \mathbf{P}\left\{\lambda^{(n)}=N\right\} \mathbf{P}\left\{\varkappa_{N}=m\right\}}{\mathbf{P}\left\{\xi^{(n)}=m\right\}} \leqslant \sqrt{n} \exp \left\{-C_{9} n^{1 / 3}(1+o(1))\right\} .
$$

Отсюда следует, что

$$
S_{4} \leqslant p_{r} \sqrt{n} \sum_{N_{4}} \frac{\mathbf{P}\left\{\lambda^{(n)}=N\right\} \mathbf{P}\left\{\varkappa_{N}=m\right\}}{\mathbf{P}\left\{\xi^{(n)}=m\right\}} \rightarrow 0 .
$$

Рассмотрим $S_{1}$. Из (5), (12), (13) и (15) находим, что

$$
\begin{aligned}
S_{1} & \leqslant \frac{n^{2} p_{r}}{k} \sum_{N_{1}}\left(\begin{array}{l}
N \\
k
\end{array}\right) p_{r}^{k}(x)\left(1-p_{r}(x)\right)^{N-k} \mathbf{P}\left\{\lambda^{(n, m)}=N\right\} \\
& \leqslant C_{10} \frac{n^{2} p_{r}}{k} \sum_{N_{1}}\left(\begin{array}{l}
N \\
k
\end{array}\right) p_{r}^{k} \exp \left\{\frac{k N}{n}\right\}\left(1-p_{r}\right)^{N-k} \mathbf{P}\left\{\lambda^{(n, m)}=N\right\} .
\end{aligned}
$$

Учитывая, что выражение $\left(\begin{array}{c}N \\ k\end{array}\right) p_{r}^{k}\left(1-p_{r}\right)^{N-k}$ возрастает при $N<k / p_{r}$, нетрудно показать, что

$$
S_{1} \leqslant C_{10} \frac{n^{3 / 2} p_{r}}{\sqrt{2 \mu}}\left(\begin{array}{c}
N_{0} \\
k
\end{array}\right)\left(1-p_{r}\right)^{N_{0}-k} p_{r}^{k} \exp \left\{k \sqrt{\frac{2 \mu}{n}}\right\} \sum_{N_{1}} \mathbf{P}\left\{\lambda^{(n, m)}=N\right\},
$$

где

$$
N_{0}=(\sqrt{2 \mu}+z) \sqrt{n}-(2 \mu)^{1 / 4} n^{7 / 12} p_{r}^{-5 / 12} \text {. }
$$

Тогда, используя нормальное приближение для биномиального распределения, находим, что $S_{1} \rightarrow 0$, что завершает доказательство теоремы 1 для первого случая.

Рассмотрим второй случай. Из (1) легко показать, что в этом случае

$$
\gamma=1+o(1)
$$

Отсюда и из (3) находим, что

$$
\begin{aligned}
\gamma p_{r} \sqrt{n} \mathbf{P}\left\{\lambda_{r}^{(n, m)}=k\right\} & =p_{r} \sqrt{n} \sum_{N=k}^{n-k r} \mathbf{P}\left\{\lambda^{(n, m)}=k\right\} \mathbf{P}\left\{\lambda_{r}(F)=k\right\}(1+o(1)) \\
& =\sum_{i=1}^{7} S_{i}(1+o(1)),
\end{aligned}
$$


где $S_{i}, i=1,2, \ldots, 7$, определены в (5), а области суммирования $N_{1}-N_{7}$ имеют вид

$$
\begin{aligned}
& N_{1}=\left\{N: k \leqslant N<\beta^{1 / 2-\delta} \sqrt{n}\right\}, \\
& N_{2}=\left\{N: \beta^{1 / 2-\delta} \sqrt{n} \leqslant N<(\sqrt{2 \mu}-A) \sqrt{, n}\right\}, \\
& N_{3}=\left\{N:(\sqrt{2 \mu}-A) \sqrt{n} \leqslant N<(\sqrt{2 \mu}+z) \sqrt{n}-(2 \mu)^{1 / 4} n^{7 / 24} p_{r}^{-5 / 12}\right\}, \\
& N_{4}=\left\{N:(\sqrt{2 \mu}+z) \sqrt{n}-(2 \mu)^{1 / 4} n^{7 / 24} p_{r}^{-5 / 12} \leqslant N \leqslant(\sqrt{2 \mu}+z) \sqrt{n}\right. \\
&\left.\quad+(2 \mu)^{1 / 4} n^{7 / 24} p_{r}^{-5 / 12}\right\}, \\
& N_{5}=\left\{N:(\sqrt{2 \mu}+z) \sqrt{n}+(2 \mu)^{1 / 4} n^{7 / 24} p_{r}^{-5 / 12}<N<\beta \sqrt{n}\right\}, \\
& N_{6}=\left\{N: \beta \sqrt{n} \leqslant N<n^{2 / 3}\right\}, \\
& N_{7}=\left\{N: n^{2 / 3} \leqslant N \leqslant n-k r\right\} ;
\end{aligned}
$$

выбор постоянных $\delta$ и $A$ будет ясен из дальнейшего.

Основной вклад в сумму, стоящую в правой части соотношения (17), вносит слагаемое $S_{4}$. Из теоремы 4 следует, что

$$
S_{4}=p_{r} \sqrt{n} \sum_{N_{4}}\left(\frac{N}{\sqrt{n}}\right)^{2 \mu} \exp \left\{-\frac{N^{2}}{2 n}+\frac{\beta}{2}\right\} \beta^{-\mu} \frac{1}{\sqrt{\pi n}} \mathbf{P}\left\{\lambda_{r}(\mathscr{F})=k\right\}(1+o(1)) .
$$

Используя соотношения (1), нетрудно получить, что при $N \in N_{4}$ выполняется соотношение

$$
S_{4}=p_{r} e^{-z^{2}} \frac{1}{\sqrt{\pi}} \sum_{N_{4}} \mathbf{P}\left\{\lambda_{r}(\mathscr{F})=k\right\}(1+o(1)) .
$$

В [3] показано (см. доказательство теоремы 5.2.2), что утверждение теоремы 6 остается верным равномерно относительно $\left(k-N p_{r}(x)\right) /\left(N p_{r}(x)\right)^{7 / 12}$ в любом конечном интервале. Используя (13), нетрудно проверить выполнение условий теоремы 6, тогда

$$
S_{4}=\frac{p_{r} e^{-z^{2}}}{\sqrt{\pi}} \sum_{N_{4}} \frac{1+o(1)}{k !}\left(N p_{r}(x)\right)^{k} \exp \left\{-N p_{r}(x)\right\} .
$$

Отсюда и из (13), используя явный вид $k$, получаем, что

$$
\begin{aligned}
S_{4} & =\frac{p_{r} e^{-z^{2}}}{\sqrt{\pi}} \sum_{N_{4}} \frac{1+o(1)}{k !}\left(N p_{r}\right)^{k} \exp \left\{-N p_{r}\right\} \\
& =\frac{p_{r} e^{-z^{2}}}{\sqrt{\pi}} \sum_{N_{4}} \frac{1+o(1)}{\left((z \gamma \sqrt{n}+\sqrt{2 \mu n}) p_{r}\right) !}\left(N p_{r}\right)^{(z \gamma \sqrt{n}+\sqrt{2 \mu n}) p_{r}} \exp \left\{-N p_{r}\right\} .
\end{aligned}
$$

Применяя формулу Стирлинга, и полагая

$$
N=(\sqrt{2 \mu}+z) \sqrt{n}+y(2 \mu n)^{1 / 4} \sqrt{\left(1+p_{r}\right) / p_{r}},
$$

находим, что

$$
S_{4}=\frac{p_{r} e^{-z^{2}}}{\pi} \sum_{y} \exp \left\{-\frac{y^{2}\left(1-p_{r}\right)}{2(1+z \gamma / \sqrt{2 \mu})}\right\} \frac{(1+o(1))}{\sqrt{2(\sqrt{2 \mu n}+z \gamma \sqrt{n}) p_{r}}},
$$


где суммирование по $y$ осуществляется в области $|y| \leqslant n^{1 / 24} p_{r}^{1 / 12} / \sqrt{1-p_{r}}$ с шагом $(2 \mu n)^{-1 / 4} \sqrt{p_{r} /\left(1-p_{r}\right)}$. Заменяя суммирование интегрированием, получаем, что

$$
S_{4}=\frac{e^{-z^{2}}}{\pi} \int_{\infty}^{\infty} e^{-t^{2}} d t(1+o(1))=\frac{e^{-z^{2}}}{\sqrt{\pi}}(1+o(1)) .
$$

Оценим сумму $S_{5}$. Согласно лемме 5.2.2 из [3],

$$
\mathbf{P}\left\{\lambda^{(n)}=N\right\}=\frac{N}{n} e^{-N^{2} /(2 n)}(1+o(1)),
$$

а из леммы 3.2 .2 из [3] следует, что

$$
\mathbf{P}\left\{\xi_{N-k}^{(r)}=n-k(r+1)\right\} \leqslant C_{11} N / n^{3 / 2} .
$$

Тогда, используя нормальное приближение для биномиального распределения, из соотношений (5), (8), (10)-(12), (14), (18), (19) получаем, что

$$
\begin{aligned}
S_{5}= & \sqrt{n} p_{r} \sum_{N_{5}} \frac{\mathbf{P}\left\{\lambda^{(n)}=N\right\} \mathbf{P}\left\{\varkappa_{N}=m\right\}}{\mathbf{P}\left\{\xi^{(n)}=m\right\}} \mathbf{P}\left\{\lambda_{r}(F)=k\right\} \\
\leqslant & C_{12} p_{r} \sqrt{n} \sum_{N_{5}} \frac{1}{\sqrt{n}} e^{-N^{2} /(2 n)}\left(\frac{\ln ((N / m) \ln (N / m))}{\ln (\alpha \ln \alpha)}\right)^{m-1} \frac{\sqrt{\beta \ln (N / m)}}{\sqrt{m}} \\
& \times \exp \left\{\frac{m}{\ln (N / m)-\beta / 2+\gamma_{1}}\right\} \beta^{-\mu}\left(2 \pi N p_{r}(x)\left(1-p_{r}(x)\right)\right)^{-1 / 2} \\
& \times \exp \left\{-\frac{\left(k-N p_{r}(x)\right)^{2}}{N p_{r}(x)\left(1-p_{r}(x)\right)}\right\} .
\end{aligned}
$$

Из (13) нетрудно видеть, что при $N \in N_{5}$

$$
\begin{aligned}
& \frac{1}{\sqrt{2 \pi N p_{r}(x)\left(1-p_{r}(x)\right)}} \exp \left\{-\frac{\left(k-N p_{r}(x)\right)^{2}}{N p_{r}(x)\left(1-p_{r}(x)\right)}\right\} \\
& \leqslant C_{13} \frac{1}{\sqrt{2 \pi N p_{r}\left(1-p_{r}\right)}} \exp \left\{-C_{14} \frac{\left(k-N p_{r}\right)^{2}}{N p_{r}\left(1-p_{r}\right)}\right\} .
\end{aligned}
$$

Кроме того, при доказательстве теоремы 5 (см. [5]) было получено, что при $y=N / \sqrt{n}$, $N \in N_{5}$

$$
\begin{aligned}
\left(\frac{\ln (N / m \ln (N / m))}{\ln (\alpha \ln (\alpha)}\right)^{m-1} & \frac{\sqrt{\beta \ln (N / m)}}{\sqrt{m}} \exp \left\{\frac{m}{\ln (N / m)}-\beta / 2\right\} \\
& =\exp \left\{\beta / 2-\frac{m \ln ^{2} y}{2 \ln ^{2}(\sqrt{n} / m)}\right\} y^{\beta(1-\ln \ln \alpha / \ln \alpha)}(1+o(1)) .
\end{aligned}
$$

Сделав необходимые преобразования, из (20)-(22) можно получить, что $S_{5} \rightarrow 0$.

Рассмотрим слагаемое $S_{6}$. Из (5), (8), (10)-(14) и леммы 3.2.2 из [3] следует, что

$$
\begin{aligned}
S_{6} \leqslant C_{15} p_{r} \sum_{N_{6}}\left(\frac{\ln (N / m \ln (N / m))}{\ln (\alpha \ln \alpha)}\right)^{m-1} & \sqrt{\frac{\beta \ln (N / m)}{m}} \exp \left\{\frac{m}{\ln (N / m)}-\frac{\beta}{2}\right\} \\
& \times \beta^{-\mu} e^{\gamma_{1}} e^{-N^{2} /(2 n)}\left(\begin{array}{l}
N \\
k
\end{array}\right) p_{r}^{k}(x)\left(1-p_{r}(x)\right)^{N-k} .
\end{aligned}
$$


При каждом фиксированном значении $p_{r}(x)$ выражение $\left(\begin{array}{c}N \\ k\end{array}\right) p_{r}^{k}(x)\left(1-p_{r}(x)\right)^{N-k}$ убывает при $N>k / p_{r}(x)$. Поэтому

$$
\begin{array}{r}
S_{6} \leqslant C_{15} p_{r} \sum_{N_{6}}\left(\frac{\ln (N / m \ln (N / m))}{\ln (\alpha \ln \alpha)}\right)^{m-1} \frac{\beta \ln (N / m)}{m} \exp \left\{\frac{m}{\ln (N / m)}-\frac{\beta}{2}+\gamma_{1}\right\} \\
\times \beta^{-m u} e^{-N^{2} /(2 n)}\left(\begin{array}{c}
N_{0} \\
k
\end{array}\right) p_{r}^{k}(x)\left(1-p_{r}(x)\right)^{N_{0}-k},
\end{array}
$$

где

$$
N_{0}=(\sqrt{2 \mu}+z) \sqrt{n}+(2 \mu)^{1 / 4} n^{7 / 24} p_{r}^{-5 / 12}
$$

Обозначая $y=N / \sqrt{n}$, нетрудно показать, что при $N \in N_{6}$

$$
\left(\frac{\ln (N / m \ln (N / m))}{\ln (\alpha \ln \alpha)}\right)^{m-1} \sqrt{\frac{\beta \ln (N / m)}{m}} \leqslant y^{\beta}, \quad \exp \left\{\frac{m}{\ln (N / m)}\right\} \leqslant e^{\beta} .
$$

Отсюда и из (23), используя нормальное приближение биномиального распределения, находим, что

$$
S_{6} \leqslant C_{15} p_{r}\left(\sqrt{2 \mu n} p_{r}\right)^{-1 / 2} \exp \left\{-C_{16} n^{1 / 12} p_{r}^{1 / 6}+\beta / 2+\gamma_{1}\right\} \beta^{-\mu} \sum_{\beta<y<n^{1 / 6}} y^{\beta} e^{-y^{2} / 2} .
$$

Нетрудно показать, что при $\beta \rightarrow \infty$

$$
\frac{1}{\sqrt{n}} \sum_{\beta<y<n^{1 / 6}} y^{\beta} e^{-y^{2} / 2} \leqslant \int_{\beta}^{\infty} \exp \left\{-C_{17} y^{2}\right\} d y \rightarrow 0 .
$$

Отсюда и из (24) следует, что $S_{6} \rightarrow 0$.

Оценим сумму $S_{7}$. При доказательстве теоремы 5 было показано (см. [5]), что

$$
\frac{\sum_{N_{7}} \mathbf{P}\left\{\lambda^{(n)}=N\right\} \mathbf{P}\left\{\varkappa_{N}=m\right\}}{\mathbf{P}\left\{\xi^{(n)}=m\right\}} \leqslant C_{18} \sqrt{n} \beta^{-\mu} \exp \left\{-\frac{n^{1 / 3}}{2}+m+\frac{\beta}{2}+\gamma_{1}\right\} .
$$

Тогда

$$
\begin{aligned}
S_{7} & =p_{r} \sqrt{n} \sum_{N_{7}} \frac{\mathbf{P}\left\{\lambda^{(n)}=N\right\} \mathbf{P}\left\{\varkappa_{N}=m\right\}}{\mathbf{P}\left\{\xi^{(n)}=m\right\}} \mathbf{P}\left\{\lambda_{r}(F)=k\right\} \\
& \leqslant p_{r} \sqrt{n} \sum_{N_{7}} \frac{\mathbf{P}\left\{\lambda^{(n)}=N\right\} \mathbf{P}\left\{\varkappa_{N}=m\right\}}{\mathbf{P}\left\{\xi^{(n)}=m\right\}} \rightarrow 0 .
\end{aligned}
$$

Рассмотрим $S_{3}$. Из теоремы 4 , соотношений (5), (12)-(14) и (18), учитывая, что выра- 
жение $\left(\begin{array}{l}N \\ k\end{array}\right) p_{r}^{k}\left(1-p_{r}\right)^{N-k}$ при $N \in N_{3}$ возрастает, нетрудно показать, что

$$
\begin{aligned}
S_{3} \leqslant & p_{r} \sqrt{n} e^{\beta / 2} \beta^{-\mu} \sum_{N_{5}} \frac{1}{\sqrt{\pi n}}\left(\frac{N}{\sqrt{n}}\right)^{2 \mu} e^{-N^{2} /(2 n)}\left(\begin{array}{c}
k \\
N
\end{array}\right) p_{r}^{k}(x)\left(1-p_{r}(x)\right)^{N-k} \\
\leqslant & C_{19} p_{r} \exp \left\{\beta / 2+C_{20} 2 \mu p_{r}\right\} \beta^{-\mu}\left(\begin{array}{c}
N_{0} \\
k
\end{array}\right) p_{r}^{k}\left(1-p_{r}\right)^{N_{0}-k} \sum_{N_{5}}\left(\frac{N}{\sqrt{n}}\right)^{2 \mu} e^{-N^{2} /(2 n)} \\
\leqslant & C_{19} p_{r} \sqrt{n} \beta^{-\mu}\left(\begin{array}{c}
N_{0} \\
k
\end{array}\right) p_{r}^{k}\left(1-p_{r}\right)^{N_{0}-k} \exp \left\{\beta / 2+C_{20} 2 \mu p_{r}\right\} \\
& \times \frac{1}{\sqrt{n}} \sum_{\sqrt{2 \mu}-A \leqslant y \leqslant \sqrt{2 \mu}+A} y^{2 \mu} e^{-y^{2} / 2},
\end{aligned}
$$

где

$$
N_{0}=(\sqrt{2 \mu}+z) \sqrt{n}-n^{7 / 24}(2 \mu)^{1 / 4} p_{r}^{-5 / 12}, \quad y=N / \sqrt{n} .
$$

Заменяя суммирование интегрированием по $t=N / \sqrt{n}-\sqrt{2 \mu}$, получаем, что при достаточно большом $A$

$$
S_{3} \leqslant C_{21} p_{r} \sqrt{n}\left(\begin{array}{c}
N_{0} \\
k
\end{array}\right) p_{r}^{k}\left(1-p_{r}\right)^{N_{0}-k} e^{C_{20} 2 \mu p_{r}} .
$$

Тогда, применяя нормальное приближение биномиального распределения, нетрудно показать, что $S_{3}$ стремится к нулю. Аналогично оценке $S_{3}$ можно получить, что $S_{2} \rightarrow 0$.

Осталось рассмотреть сумму $S_{1}$. Если $\beta \asymp \ln n$, то, применяя результаты, полученные при доказательстве теоремы 5 (см. [5]), легко найти, что при достаточно малом $\delta$

$$
S_{1} \leqslant p_{r} \sqrt{n} \frac{\sum_{N_{1}} \mathbf{P}\left\{\lambda^{(n)}=N\right\} \mathbf{P}\left\{\varkappa_{N}=m\right\}}{\mathbf{P}\left\{\xi^{(n)}=m\right\}} \leqslant \exp \left\{\ln \left(\sqrt{n} p_{r}\right)-C_{22} \beta \ln \beta\right\} \rightarrow 0 .
$$

Если $\beta=o(\ln n)$, то разобьем область суммирования $N_{1}$ на три части:

$$
\begin{aligned}
& N_{1}^{(1)}=\left\{N: k \leqslant N<\varepsilon_{1} \sqrt{n}\right\}, \\
& N_{1}^{(2)}=\left\{N: \varepsilon_{1} \sqrt{n} \leqslant N<(\ln \beta) \sqrt{n}\right\}, \\
& N_{1}^{(3)}=\left\{N:(\ln \beta) \sqrt{n} \leqslant N<\beta^{1 / 2-\delta} \sqrt{n}\right\},
\end{aligned}
$$

выбор положительной постоянной $\varepsilon_{1}$ будет ясен из дальнейшего, а соответствующие суммы обозначим через $S_{1}^{(1)}, S_{1}^{(2)}$ и $S_{1}^{(3)}$.

Из соотношений (5), (12)-(14) и (19) нетрудно найти, что

$$
\begin{aligned}
S_{1}^{(3)} & \leqslant C_{23} p_{r} \sqrt{n} \sum_{N_{1}^{(3)}} \mathbf{P}\left\{\lambda^{(n, m)}=N\right\}\left(\begin{array}{l}
N \\
k
\end{array}\right) p_{r}^{k}(x)\left(1-p_{r}(x)\right)^{N-k} \\
& \leqslant C_{24} p_{r} \sqrt{n} \sum_{N_{1}^{(3)}} \mathbf{P}\left\{\lambda^{(n, m)}=N\right\}\left(\begin{array}{l}
N \\
k
\end{array}\right) p_{r}^{k} \exp \{k N / n\}\left(1-p_{r}\right)^{N-k} .
\end{aligned}
$$


Поскольку выражение $\left(\begin{array}{c}N \\ k\end{array}\right) p_{r}^{k}\left(1-p_{r}\right)^{N-k}$ возрастает при $N<k / p_{r}$, то при достаточно малом $\delta$

$$
S_{1}^{(3)} \leqslant C_{25} \sqrt{n} p_{r}\left(\begin{array}{c}
N_{0} \\
k
\end{array}\right) p_{r}^{k}\left(1-p_{r}\right)^{N_{0}-k} \exp \left\{C_{26} \mu p_{r}\right\}
$$

где

$$
N_{0}=(\sqrt{2 \mu}+z) \sqrt{n}-(2 \mu)^{1 / 4} n^{7 / 24} p_{r}^{-5 / 12} .
$$

Тогда, используя нормальное приближение биномиального распределения, легко получить, что $S_{1}^{(3)} \rightarrow 0$.

Следуя рассуждениям, приведенным для оценки $S_{1}^{(3)}$, нетрудно показать, что при достаточно малом $\varepsilon_{1}$ суммы $S_{1}^{(2)}$ и $S_{1}^{(1)}$ стремятся к нулю, при этом для оценки $S_{1}^{(2)}$ применяют лемму 3 из [1], а для $S_{1}^{(1)}$ - леммы 3.3.1, 3.3.2, 2.3.3 и 2.3.4 из [3], что и завершает доказательство теоремы 1 для второго случая.

Рассмотрим третий случай. Вероятность $\mathbf{P}\left\{\lambda_{r}^{(n, m)}=k\right\}$ можно представить в виде суммы (17), где $S_{i}, i=1, \ldots 7$, определены в (5), а

$$
\begin{aligned}
& N_{1}=\left\{N: k \leqslant N<\beta^{1 / 2-\delta} \sqrt{n}\right\}, \\
& N_{2}=\left\{N: \beta^{1 / 2-\delta} \sqrt{n} \leqslant N<(\sqrt{2 \mu}-A) \sqrt{n}\right\}, \\
& N_{3}=\left\{N:(\sqrt{2 \mu}-A) \sqrt{n} \leqslant N<(\sqrt{2 \mu}+z) \sqrt{n}-n^{7 / 24} p_{r}^{-5 / 12}(2 \mu)^{1 / 4}(\ln \mu)^{-1}\right\}, \\
& N_{4}=\left\{N:(\sqrt{2 \mu}+z) \sqrt{n}-n^{7 / 24} p_{r}^{-5 / 12}(2 \mu)^{1 / 4}(\ln \mu)^{-1} \leqslant N \leqslant(\sqrt{2 \mu}+z) \sqrt{n}\right. \\
&\left.\quad+n^{7 / 24} p_{r}^{-5 / 12}(2 \mu)^{1 / 4}(\ln \mu)^{-1}\right\}, \\
& N_{5}=\left\{N:(\sqrt{2 \mu}+z) \sqrt{n}+n^{7 / 24} p_{r}^{-5 / 12}(2 \mu)^{1 / 4}(\ln \mu)^{-1}<N<\beta \sqrt{n}\right\}, \\
& N_{6}=\left\{N: \beta \sqrt{n} \leqslant N<n^{2 / 3}\right\}, \\
& N_{7}=\left\{N: n^{2 / 3} \leqslant N \leqslant n-k r\right\}
\end{aligned}
$$

выбор положительных постоянных $\delta$ и $A$ будет ясен из дальнейшего.

Основной вклад в сумму, стоящую в правой части соотношения (17), вносит слагаемое $S_{4}$. Нетрудно показать, что при $N \in N_{4}$ можно использовать теоремы 4 и 6 . Аналогично тому, как это было сделано для суммы $S_{4}$ во втором случае, находим, что

$$
S_{4}=\frac{p_{r} e^{-z^{2}}}{\pi} \sum_{y} \exp \left\{-\frac{y^{2}\left(1-p_{r}\right)}{2(1+z \gamma / \sqrt{2 \mu})}\right\} \frac{1}{\sqrt{2 p_{r}(\sqrt{2 \mu}+z \gamma) \sqrt{n}}}(1+o(1)) \text {; }
$$

суммирование в последней сумме осуществляется с шагом $(2 \mu n)^{-1 / 4} \sqrt{p_{r} /\left(1-p_{r}\right)}$ по области $|y| \leqslant n^{1 / 24} p_{r}^{1 / 12} /\left(\sqrt{1-p_{r}} \ln \mu\right)$. Заменяя суммирование интегрированием, получаем, что

$$
S_{4}=e^{-z^{2}} \frac{(1+o(1))}{\sqrt{\pi}} .
$$

Оценка сумм $S_{1}, S_{2}, S_{5}-S_{7}$ проводится аналогично оценкам $S_{1}, S_{2}, S_{5}-S_{7}$, приведенным в доказательстве второго случая.

Рассмотрим $S_{3}$. Рассуждая так же, как и при оценивании $S_{3}$ в предыдущем случае, находим, что при достаточно большом $A$

$$
S_{3} \leqslant C_{27} p_{r} \sqrt{n}\left(\begin{array}{c}
N_{0} \\
k
\end{array}\right) p_{r}^{k}\left(1-p_{r}\right)^{N_{0}-k}
$$


где

$$
N_{0}=(\sqrt{2 \mu}+z) \sqrt{n}-n^{7 / 24}(2 \mu)^{1 / 4} p_{r}^{-5 / 12} \ln ^{-1} \mu .
$$

Отсюда, используя нормальное приближение биномиального распределения, легко получить, что $S_{3} \rightarrow 0$, значит, и для третьего случая утверждение теоремы 1 доказано.

Докажем справедливость теоремы 1 для четвертого случая. Учитывая, что в этом случае $\gamma=1+o(1)$, вероятность $\mathbf{P}\left\{\lambda_{r}^{(n, m)}=k\right\}$ можно представить в виде

$$
\gamma p_{r} \sqrt{n} \mathbf{P}\left\{\lambda_{r}^{(n, m)}=k\right\}=\sum_{i=1}^{6} S_{i}(1+o(1)),
$$

где $S_{i}, i=1, \ldots, 6$, определены в (5), а

$$
\begin{aligned}
N_{1}=\left\{N: \beta^{1 / 2-\delta} \sqrt{n} \leqslant N<\sqrt{2 \mu n}+z \sqrt{n}-(2 \mu n)^{7 / 24} p_{r}^{-5 / 12}\right\}, \\
N_{2}=\left\{N: \sqrt{2 \mu n}+z \sqrt{n}-(2 \mu n)^{7 / 24} p_{r}^{-5 / 12} \leqslant N<\sqrt{2 \mu n}+z \sqrt{n}\right. \\
\left.\quad-(2 \mu)^{5 / 24} n^{7 / 24} p_{r}^{-5 / 12}\right\}, \\
N_{3}=\left\{N: \sqrt{2 \mu n}+z \sqrt{n}-(2 \mu)^{5 / 24} n^{7 / 24} p_{r}^{-5 / 12} \leqslant N \leqslant \sqrt{2 \mu n}+z \sqrt{n}\right. \\
\left.\quad+(2 \mu)^{5 / 24} n^{7 / 24} p_{r}^{-5 / 12}\right\}, \\
N_{4}=\left\{N: \sqrt{2 \mu n}+z \sqrt{n}+(2 \mu)^{5 / 24} n^{7 / 24} p_{r}^{-5 / 12}<N \leqslant \sqrt{2 \mu n}+z \sqrt{n}\right. \\
\left.\quad+(2 \mu n)^{7 / 24} p_{r}^{-5 / 12}\right\}, \\
N_{5}=\left\{N: \sqrt{2 \mu n}+z \sqrt{n}+(2 \mu n)^{7 / 24} p_{r}^{-5 / 12}<N \leqslant \beta^{1 / 2+\delta} \sqrt{n}\right\}, \\
N_{6}=\left\{N: N \notin N_{i}, i=1, \ldots, 5\right\} ;
\end{aligned}
$$

выбор положительной постоянной $\delta$ будет ясен из дальнейшего.

Основной вклад в сумму, стоящую в правой части равенства (25), вносит слагаемое $S_{3}$. Из теорем 4 и 7 , соотношений (1), (5) и (13) можно получить, что

$$
\begin{aligned}
S_{3} & =p_{r} \sqrt{n} \sum_{N_{3}} \mathbf{P}\left\{\lambda^{(n, m)}=N\right\} \mathbf{P}\left\{\lambda_{r}(\mathscr{F})=k\right\} \\
& =p_{r} \sqrt{n} \sum_{N_{3}} \frac{1}{k !}\left(N p_{r}(x)\right)^{k} \exp \left\{-N p_{r}(x)\right\} \frac{(N / \sqrt{n})^{2 \mu} e^{-N^{2} /(2 n)} \beta^{-\mu} e^{\beta / 2}}{\sqrt{\pi n}}(1+o(1)) \\
& =p_{r} e^{-z^{2}} \frac{1+o(1)}{\sqrt{\pi}} \sum_{N_{3}} \frac{1}{k !}\left(N p_{r}\right)^{k} \exp \left\{-N p_{r}\right\} .
\end{aligned}
$$

Положим

$$
N=(\sqrt{2 \mu}+z) \sqrt{n}+y(2 \mu n)^{1 / 4} \sqrt{\left(1-p_{r}\right) / p_{r}} .
$$

Используя явный вид $k$ и формулу Стирлинга, из (26) находим, что

$$
S_{3}=p_{r} e^{-z^{2}} \frac{1+o(1)}{\sqrt{\pi}} \sum_{y} \exp \left\{-\frac{y^{2}\left(1-p_{r}\right)}{2(1+z \gamma / \sqrt{2 \mu})}\right\} \frac{1}{\sqrt{2 p_{r}(\sqrt{2 \mu}+z \gamma) \sqrt{n}}},
$$


где суммирование по $y$ осуществляется в области $|y| \leqslant n^{1 / 24}(2 \mu)^{1 / 24} p_{r}^{1 / 12}$ с шагом $(2 \mu n)^{1 / 4} \sqrt{p_{r} /\left(1-p_{r}\right)}$. Заменяя суммирование интегрированием, получаем, что

$$
S_{3}=e^{-z^{2}} \frac{1+o(1)}{\sqrt{\pi}} .
$$

Нетрудно проверить, что в области $N_{2}$ условия теоремы 6 выполнены. Из этой теоремы и соотношений (5), (13), учитывая, что функщия $f(N)=\left(N p_{r}\right)^{k} \exp \left\{-N p_{r}\right\}$ в области $N_{2}$ возрастает, несложно найти следующую оценку для $S_{2}$ :

$$
\begin{aligned}
S_{2} & =p_{r} \sqrt{n} \sum_{N_{2}} \mathbf{P}\left\{\lambda^{(n, m)}=N\right\} \frac{1}{k !}\left(N p_{r}\right)^{k} \exp \left\{-N p_{r}\right\}(1+o(1)) \\
& \leqslant C_{28}\left(\frac{p_{r} \sqrt{n}}{2 \pi \sqrt{2 \mu}}\right)^{1 / 2} \exp \left\{-\frac{1}{2}(2 \mu)^{-1 / 12} n^{1 / 12} p_{r}^{1 / 6}\right\} \rightarrow 0,
\end{aligned}
$$

поскольку, согласно условиям теоремы, $p_{r} \sqrt{n /(2 \mu)} \rightarrow \infty$.

Оценка суммы $S_{1}$ проводится аналогично оценке $S_{2}$, с использованием того, что функция $f(N)$ в области $N_{1}$ убывает.

Оценим сумму $S_{4}$. Из теорем 4 и 6 и соотношений (5), (13) нетрудно получить, что при выполнении условий теоремы

$$
\begin{aligned}
S_{4} & =p_{r} \sqrt{n} \sum_{N_{4}} \mathbf{P}\left\{\lambda^{(n, m)}=N\right\} \frac{1}{k !}\left(N p_{r}(x)\right)^{k} \exp \left\{-N p_{r}(x)\right\}(1+o(1)) \\
& =p_{r} \sqrt{n} \sum_{N_{4}} \mathbf{P}\left\{\lambda^{(n, m)}=N\right\} \frac{1}{k !}\left(N p_{r}\right)^{k} \exp \left\{-N p_{r}\right\}(1+o(1)) .
\end{aligned}
$$

Отсюда, используя формулу Стирлинга, находим, что

$$
\begin{aligned}
S_{4} & \leqslant p_{r} \sqrt{n}(k !)^{-1}\left(k+(2 \mu)^{5 / 24} n^{7 / 24} p_{r}^{7 / 12}\right)^{k} \exp \left\{-\left(k+(2 \mu)^{5 / 24} n^{7 / 24} p_{r}^{7 / 12}\right)\right\} \\
& \leqslant \sqrt{\frac{b}{2 \pi}} \exp \left\{\frac{b^{1 / 6}}{2}(1+o(1))\right\} \rightarrow 0 .
\end{aligned}
$$

Рассмотрим слагаемое $S_{5}$. Из соотношений (5), (12)-(14) и леммы 3.2 .2 из [3] следует, что

$$
\begin{aligned}
S_{5} & \leqslant p_{r} \sqrt{n} \sum_{N_{5}} \mathbf{P}\left\{\lambda^{(n)}=N\right\}\left(\begin{array}{l}
N \\
k
\end{array}\right) p_{r}^{k}(x)\left(1-p_{r}(x)\right)^{N-k} \\
& \leqslant C_{27} p_{r} \sqrt{n} \sum_{N_{5}} \mathbf{P}\left\{\lambda^{(n, m)}=N\right\}\left(\begin{array}{l}
N \\
k
\end{array}\right) p_{r}^{k} \exp \{k N / n\}\left(1-p_{r}\right)^{N-k} .
\end{aligned}
$$

Выражение $\left(\begin{array}{l}N \\ k\end{array}\right) p_{r}^{k}\left(1-p_{r}\right)^{N-k}$ при $N>k / p_{r}$ убывает. Тогда, применяя нормальное приближение биномиального распределения, получаем, что

$$
\begin{aligned}
S_{5} & \leqslant C_{29} p_{r} \sqrt{n}\left(\begin{array}{c}
N_{0} \\
k
\end{array}\right) p_{r}^{k}\left(1-p_{r}\right)^{N_{0}-k} \exp \left\{C_{30} \beta^{1+\delta} p_{r}\right\} \\
& \leqslant C_{31}\left(p_{r} \sqrt{n /(2 \mu)}\right)^{1 / 2} \exp \left\{-C_{31}(\mu n)^{1 / 12} p_{r}^{1 / 6}\right\} \rightarrow 0 .
\end{aligned}
$$


Осталось рассмотреть сумму $S_{6}$ :

$$
\begin{aligned}
S_{6} & =p_{r} \sqrt{n} \sum_{N_{6}} \mathbf{P}\left\{\lambda^{(n, m)}=N\right\} \mathbf{P}\left\{\lambda_{r}(F)=k\right\} \leqslant \mu^{9 / 5} \sum_{N_{6}} \mathbf{P}\left\{\lambda^{(n, m)}=N\right\} \\
& =\mu^{9 / 5} \frac{\sum_{N_{6}} \mathbf{P}\left\{\lambda^{(n)}=N\right\} \mathbf{P}\left\{\varkappa_{N}=m\right\}}{\mathbf{P}\left\{\xi^{(n)}=m\right\}} .
\end{aligned}
$$

В доказательстве теоремы 5 (см. [5]) показано, что при достаточно малом $\delta$

$$
\frac{\sum_{N_{6}} \mathbf{P}\left\{\lambda^{(n)}=N\right\} \mathbf{P}\left\{\varkappa_{N}=m\right\}}{\mathbf{P}\left\{\xi^{(n)}=m\right\}} \leqslant \sqrt{n} \exp \left\{-C_{31} n^{1 / 3}\right\} .
$$

Отсюда и из (27) следует, что $S_{6} \rightarrow 0$. Значит, и для четвертого случая утверждение теоремы 1 остается верным.

Докажем справедливость теоремы 1 для пятого случая. Представим вероятность $\mathbf{P}\left\{\lambda_{r}^{(n, m)}=k\right\}$ в виде суммы (25), где

$$
\begin{aligned}
& S_{i}=\gamma p_{r} \sqrt{n} \sum_{N_{i}} \mathbf{P}\left\{\lambda^{(n, m)}=N\right\} \mathbf{P}\left\{\lambda_{r}(\mathscr{F})=k\right\}, \quad i=1, \ldots, 6, \\
& N_{1}=\left\{N: \beta^{1 / 2-\delta} \sqrt{n} \leqslant N<\sqrt{2 \mu n}-(2 \mu n)^{7 / 24} p_{r}^{-5 / 12}\right\}, \\
& N_{2}=\left\{N: \sqrt{2 \mu n}-(2 \mu n)^{7 / 24} p_{r}^{-5 / 12} \leqslant N<\sqrt{2 \mu n}-A \sqrt{n}\right\}, \\
& N_{3}=\{N: \sqrt{2 \mu n}-A \sqrt{n} \leqslant N \leqslant \sqrt{2 \mu n}+A \sqrt{n}\}, \\
& N_{4}=\left\{N: \sqrt{2 \mu n}+A \sqrt{n}<N<\sqrt{2 \mu n}+(2 \mu n)^{7 / 24} p_{r}^{-5 / 12}\right\}, \\
& N_{5}=\left\{N: \sqrt{2 \mu n}+(2 \mu n)^{7 / 24} p_{r}^{-5 / 12}<N \leqslant \beta^{1 / 2+\delta} \sqrt{n}\right\}, \\
& N_{6}=\left\{N: N \notin N_{i}, i=1, \ldots, 5\right\} ;
\end{aligned}
$$

выбор положительной постоянной $A$ будет ясен из дальнейшего.

Основной вклад в сумму, стоящую в правой части соотношения (25), дает слагаемое $S_{3}$.

Положим $y=N / \sqrt{n}$. Из теорем 4 и 6 и соотношений (1), (13), (28) нетрудно получить, что

$$
S_{3}=\frac{\left(p_{r} \sqrt{n}\right)^{k} p_{r} \beta^{-\mu} e^{\beta / 2} \gamma}{k ! \sqrt{\pi}} \sum_{|y-\sqrt{2 \mu}| \leqslant A} \exp \left\{-\frac{y^{2}}{2}-y p_{r} \sqrt{n}\right\} y^{2 \mu+k}(1+o(1)) .
$$

Отсюда видно, что при достаточно большом $A$

$$
S_{3}=e^{-z^{2}} \frac{1+o(1)}{\sqrt{\pi}} .
$$

Из соотношений (12)-(14) и леммы 3.2.2 [3] находим, что

$$
\begin{aligned}
S_{1} & =\gamma p_{r} \sqrt{n} \sum_{N_{1}} \mathbf{P}\left\{\lambda^{(n)}=N\right\} \mathbf{P}\left\{\lambda_{r}(F)=k\right\} \\
& \leqslant C_{34} \gamma p_{r} \sqrt{n} \sum_{N_{1}} \mathbf{P}\left\{\lambda^{(n)}=N\right\}\left(\begin{array}{l}
N \\
k
\end{array}\right)\left(p_{r}(x)\right)^{k}\left(1-p_{r}(x)\right)^{N-k} \\
& \leqslant C_{34} \gamma p_{r} \sqrt{n} \sum_{N_{1}} \mathbf{P}\left\{\lambda^{(n, m)}=N\right\}\left(\begin{array}{l}
N \\
k
\end{array}\right) p_{r}^{k} \exp \{k N / n\}\left(1-p_{r}\right)^{N-k} .
\end{aligned}
$$


Положим

$$
N_{0}=\sqrt{2 \mu n}-(2 \mu n)^{7 / 24} p_{r}^{-5 / 12} \text {. }
$$

Выражение $\left(\begin{array}{l}N \\ k\end{array}\right) p_{r}^{k}\left(1-p_{r}\right)^{N-k}$ возрастает при $N \in N_{1}$. Учитывая, что в рассматриваемом случае $\gamma$ ограничено, и используя нормальное приближение биномиального распределения, находим, что

$$
\begin{aligned}
S_{1} & \leqslant C_{35} p_{r} \sqrt{n}\left(\begin{array}{c}
N_{0} \\
k
\end{array}\right) p_{r}^{k}\left(1-p_{r}\right)^{N_{0}-k} \exp \left\{C_{36} \mu p_{r}\right\} \\
& \leqslant C_{35}\left(\frac{p_{r} \sqrt{n}}{\sqrt{2 \mu}}\right)^{1 / 2} \exp \left\{-C_{37}\left(\sqrt{2 \mu n} p_{r}\right)^{1 / 6}\right\} \rightarrow 0 .
\end{aligned}
$$

Из теоремы 6, соотношений (8), (13), (28), учитывая, что $\gamma$ ограничено, а функщия $f(x)=x^{k} e^{-x}$ возрастает при $x<k$, получаем оценку

$$
\begin{aligned}
S_{2} & =\gamma p_{r} \sqrt{n} \sum_{N_{2}} \mathbf{P}\left\{\lambda^{(n, m)}=N\right\} \frac{1}{k !}\left(N p_{r}\right)^{k} \exp \left\{-N p_{r}\right\}(1+o(1)) \\
& \leqslant \frac{\gamma p_{r} \sqrt{n}}{\sqrt{2 \pi k}} \exp \left\{-\frac{A^{2}\left(p_{r} \sqrt{n}\right)^{2}}{2 k}\right\} \sum_{N_{2}} \mathbf{P}\left\{\lambda^{(n, m)}=N\right\} \\
& =\frac{\gamma p_{r} \sqrt{n}}{\sqrt{2 \pi k}} \exp \left\{-\frac{A^{2}\left(p_{r} \sqrt{n}\right)^{2}}{2 k}\right\} \frac{\sum_{N_{2}} \mathbf{P}\left\{\lambda^{(n)}=N\right\} \mathbf{P}\left\{\varkappa_{N}=m\right\}}{\mathbf{P}\left\{\xi^{(n)}=m\right\}} .
\end{aligned}
$$

При доказательстве теоремы 5 (см. [5]) было показано, что

$$
\frac{\sum_{N_{2}} \mathbf{P}\left\{\lambda^{(n)}=N\right\} \mathbf{P}\left\{\varkappa_{N}=m\right\}}{\mathbf{P}\left\{\xi^{(n)}=m\right\}}=o(1) .
$$

Отсюда и из (29) получаем, что $S_{2} \rightarrow 0$.

Оценка суммы $S_{4}$ проводится аналогично оценке $S_{2}$, учитывая, что в области $N_{4}$ функция $f(N)=\left(N p_{r}\right)^{k} e^{-N p_{r}}$ убывает. Оценки $S_{5}$ и $S_{6}$ совпадают с оценками $S_{5}$ и $S_{6}$, соответственно, полученными при доказательстве четвертого случая. Следовательно, и для пятого случая утверждение теоремы 1 справедливо.

Докажем справедливость теоремы 1 для шестого случая. Вероятность $\mathbf{P}\left\{\lambda_{r}^{(n, m)}=k\right\}$ можно представить в виде (25), где $S_{i}, i=1, \ldots, 6$, определены в (28), а

$$
\begin{aligned}
& N_{1}=\left\{N: \beta^{1 / 2+\delta} \sqrt{n} \leqslant N<\sqrt{2 \mu n}-(2 \mu n)^{7 / 24} p_{r}^{-5 / 12}\right\}, \\
& N_{2}=\left\{N: \sqrt{2 \nu n}-(2 \mu n)^{7 / 24} \leqslant N<\sqrt{2 \mu n}-A \sqrt{n}\right\}, \\
& N_{3}=\{N: \sqrt{2 \mu n}-A \sqrt{n} \leqslant N \leqslant \sqrt{2 \mu n}+A \sqrt{n}\}, \\
& N_{4}=\left\{N: \sqrt{2 \mu m}+A \sqrt{n}<N \leqslant \sqrt{2 \mu n}+(2 \mu n)^{7 / 24} p_{r}^{-5 / 12}\right\}, \\
& N_{5}=\left\{N: \sqrt{2 \mu n}+(2 \mu n)^{7 / 24} p_{r}^{-5 / 12}<N \leqslant \beta^{1 / 2+\delta} \sqrt{n}\right\}, \\
& N_{6}=\left\{N: N \notin N_{i}, i=1, \ldots, 5\right\} ;
\end{aligned}
$$

выбор положительной постоянной $A$ будет ясен из дальнейшего. 
Рассмотрим сумму $S_{3}$. Из теорем 4 и 6 и соотношения (13), получаем,что

$$
\begin{aligned}
S_{3} & =\gamma p_{r} \sqrt{n} \sum_{N_{3}} \frac{1}{k !}\left(N p_{r}(x)\right)^{k} \exp \left\{-N p_{r}(x)\right\}\left(\frac{N}{\sqrt{n}}\right)^{2 \mu} e^{-N^{2} /(2 n)} \beta^{-\mu} e^{\beta / 2} \frac{1+o(1)}{\sqrt{\pi n}} \\
& =\gamma p_{r} \sqrt{n} \sum_{N_{3}} \frac{1}{k !}\left(N p_{r}\right)^{k} \exp \left\{-N p_{r}-\frac{N^{2}}{2 n}+\frac{\beta}{2}\right\}\left(\frac{N}{\sqrt{n}}\right)^{2 \mu} \beta^{-\mu} \frac{1+o(1)}{\sqrt{\pi n}} .
\end{aligned}
$$

Полагая

$$
t=N / \sqrt{n}-\sqrt{2 \mu}
$$

нетрудно найти, что

$$
S_{3}=\gamma\left(p_{r} \sqrt{n}\right)^{k} p_{r} \beta^{-\mu} \exp \left\{-\mu \sqrt{2 \mu n} p_{r}+\beta / 2\right\}(2 \mu)^{\mu+k / 2} \frac{1}{k !} \sum_{|t| \leqslant A} \exp \left\{-t^{2}\right\}(1+o(1)) .
$$

Суммирование в последней сумме осуществляется с шагом $1 / \sqrt{n}$. Переходя от суммирования к интегрированию, получаем, что при достаточно большом $A$

$$
S_{3}=\frac{1}{\sqrt{\pi}} e^{-z^{2}}(1+o(1)) .
$$

Оценим $S_{1}$. Из (12), (13) и (28) следует, что

$$
\begin{aligned}
S_{1} & =\gamma p_{r} \sqrt{n} \sum_{N_{1}} \mathbf{P}\left\{\lambda^{(n, m)}=N\right\} \mathbf{P}\left\{\lambda_{r}(\mathscr{F})=k\right\} \\
& \leqslant C_{38} \gamma p_{r} \sqrt{n} \exp \left\{C_{39} \frac{2 \mu n p_{r}}{n}\right\} \sum_{N_{1}} \mathbf{P}\left\{\lambda^{(n, m)}=N\right\}\left(\begin{array}{l}
N \\
k
\end{array}\right) p_{r}^{k}\left(1-p_{r}\right)^{N-k} .
\end{aligned}
$$

Выражение $\left(\begin{array}{c}N \\ k\end{array}\right) p_{r}^{k}\left(1-p_{r}\right)^{N-k}$ при $N \in N_{1}$ возрастает. Положим

$$
N_{0}=\sqrt{2 \mu n}-(2 \mu n)^{7 / 24} p_{r}^{-5 / 12} \text {. }
$$

Тогда

$$
\begin{aligned}
S_{1} & \leqslant C_{38} \gamma p_{r} \sqrt{n} \exp \left\{C_{39} 2 \mu p_{r}\right\} \sum_{N_{1}} \mathbf{P}\left\{\lambda^{(n, m)}=N\right\}\left(\begin{array}{c}
N_{0} \\
k
\end{array}\right) p_{r}^{k}\left(1-p_{r}\right)^{N_{0}-k} \\
& \leqslant C_{38} \gamma p_{r} \sqrt{n}\left(\begin{array}{c}
N_{0} \\
k
\end{array}\right) p_{r}^{k}\left(1-p_{r}\right)^{N_{0}-k} \exp \left\{C_{39} 2 \mu p_{r}\right\} .
\end{aligned}
$$

Отсюда, используя нормальное приближение биномиального распределения, легко показать, что $S_{1} \rightarrow 0$.

Суммы $S_{2}$ и $S_{4}$ оцениваются так же, как $S_{2}$ в доказательстве четвертого случая. Функщия $f(x)=x^{k} e^{-x}$ достигает максимального значения при $x=k$. Тогда, используя формулу Стирлинга, из соотношений (1), (8), (13), (28) и теоремы 6 находим, что

$$
\begin{aligned}
S_{2}+S_{4} & =\gamma p_{r} \sqrt{n} \sum_{N_{2} \cup N_{4}} \mathbf{P}\left\{\lambda^{(n, m)}=N\right\} \frac{1}{k !}\left(N p_{r}\right)^{k} e^{-N p_{r}}(1+o(1)) \\
& \leqslant \sum_{N_{2} \cup N_{4}} \frac{\mathbf{P}\left\{\lambda^{(n)}=N\right\} \mathbf{P}\left\{\varkappa_{N}=m\right\}}{\mathbf{P}\left\{\xi^{(n)}=m\right\}} .
\end{aligned}
$$


При доказательстве теоремы 5 (см. [5]) показано, что

$$
\frac{\sum_{N_{2} \cup N_{4}} \mathbf{P}\left\{\lambda^{(n)}=N\right\} \mathbf{P}\left\{\varkappa_{N}=m\right\}}{\mathbf{P}\left\{\xi^{(n)}=m\right\}}=o(1) .
$$

Следовательно, $S_{2}+S_{4} \rightarrow 0$.

Рассмотрим $S_{5}$. Из (1), (8), (12)-(14), (28) и леммы 3.2 .2 из [3], используя тот факт, что при $N \in N_{5}$ выражение $\left(\begin{array}{l}N \\ k\end{array}\right) p_{r}^{k}\left(1-p_{r}\right)^{N-k}$ убывает, находим, что

$$
\begin{aligned}
S_{5} & =\gamma p_{r} \sqrt{n} \sum_{N_{5}} \mathbf{P}\left\{\lambda^{(n, m)}=N\right\} \mathbf{P}\left\{\lambda_{r}(F)=k\right\} \\
& \leqslant C_{40} \frac{p_{r} \sqrt{n}}{\sqrt{b}} \sum_{N_{5}} \mathbf{P}\left\{\lambda^{(n, m)}=N\right\}\left(\begin{array}{c}
N \\
k
\end{array}\right) p_{r}^{k}(x)\left(1-p_{r}(x)\right)^{N-k} \\
& \leqslant C_{40} \frac{p_{r} \sqrt{n}}{\sqrt{b}} \exp \left\{C_{38} \beta^{1+\delta} p_{r}\right\} \sum_{N_{5}}\left(\begin{array}{c}
N_{0} \\
k
\end{array}\right) p_{r}^{k}\left(1-p_{r}\right)^{N-k} \mathbf{P}\left\{\lambda^{(N, m)}=N\right\},
\end{aligned}
$$

где

$$
N_{0}=\sqrt{2 \mu n}+(2 \mu n)^{7 / 24} p_{r}^{-5 / 12} .
$$

Тогда, используя нормальное приближение биномиального распределения, легко показать, что $S_{5} \rightarrow 0$.

Сумма $S_{6}$ оценивается так же, как $S_{6}$ при доказательстве четвертого случая теоремы 1. Теперь теорема 1 доказана полностью.

Пусть выполнены условия теоремы 2. Вероятность $\mathbf{P}\left\{\lambda_{r}^{(n, m)}=k\right\}$ можно представить в виде суммы

$$
\mathbf{P}\left\{\lambda_{r}^{(n, m)}=k\right\}=\sum_{N=m}^{n-k r} \mathbf{P}\left\{\lambda^{(n, m)}=N\right\} \mathbf{P}\left\{\lambda_{r}(F)=k\right\}=S_{1}+S_{2},
$$

где

$$
\begin{array}{rlrl}
S_{i} & =\sum_{N_{i}} \mathbf{P}\left\{\lambda^{(n, m)}=N\right\} \mathbf{P}\left\{\lambda_{r}(F)=k\right\}, & i & =1,2, \\
N_{1} & =\{N: \sqrt{2 \mu n}-A \sqrt{n} \leqslant N \leqslant \sqrt{2 \mu n}+A \sqrt{n}\}, & N_{2}=\left\{N: N \notin N_{1}\right\} ;
\end{array}
$$

выбор положительной постоянной $A$ будет ясен из дальнейшего.

Рассмотрим сумму $S_{1}$. Из теорем 4,6 и соотношений (13), (30) получаем, что при выполнении условий теоремы 2

$$
\begin{aligned}
S_{1} & =\sum_{N_{1}} \frac{1}{k !}\left(N p_{r}(x)\right)^{k} \exp \left\{-N p_{r}(x)\right\} \mathbf{P}\left\{\lambda^{(n, m)}=N\right\}(1+o(1)) \\
& =\sum_{N_{1}} \frac{1}{k !}\left(N p_{r}\right)^{k} \exp \left\{-N p_{r}\right\} \mathbf{P}\left\{\lambda^{(n, m)}=N\right\}(1+o(1)) \\
& =\frac{1}{k !} \theta^{k} e^{-\theta} \sum_{N_{1}} \mathbf{P}\left\{\lambda^{(n, m)}=N\right\}(1+o(1)) \\
& =\frac{1+o(1)}{k !} \theta^{k} e^{-\theta+\beta / 2} \beta^{-\mu} \frac{1}{\sqrt{\pi n}} \sum_{N_{1}}\left(\frac{N}{\sqrt{n}}\right)^{2 \mu} \exp \left\{-\frac{N^{2}}{2 n}\right\} .
\end{aligned}
$$


Обозначим $t=N / \sqrt{n}-\sqrt{2 \mu}$; нетрудно показать, что

$$
S_{1}=\frac{1}{k !} \theta^{k} e^{-\theta} \frac{1}{\sqrt{\pi n}} \sum_{-A \leqslant t \leqslant A} \exp \left\{-t^{2}\right\}(1+o(1)) .
$$

Заменяя суммирование интегрированием, находим, что при достаточно большом $A$

$$
S_{1}=\frac{1}{k !} \theta^{k} e^{-\theta}(1+o(1)) \text {. }
$$

Нетрудно показать, что $S_{2}=o(1)$. Действительно,

$$
S_{2} \leqslant \sum_{N_{2}} \mathbf{P}\left\{\lambda^{(n, m)}=N\right\}=\frac{\sum_{N_{2}} \mathbf{P}\left\{\lambda^{(n)}=N\right\} \mathbf{P}\left\{\varkappa_{N}=m\right\}}{\mathbf{P}\left\{\xi^{(n)}=m\right\}}=o(1) .
$$

Последнее соотношение было получено при доказательстве теоремы 5 (см. [5]). Отсюда и из (32) следует справедливость теоремы 2.

Пусть выполнены условия теоремы 3. Вероятность $\mathbf{P}\left\{\lambda_{r}^{(n, m)}=k\right\}$ можно представить в виде (30), где $S_{i}, i=1,2$, определены в (31).

Из теорем 4 и 6 находим, что при $k=0$

$$
S_{1}=(1+o(1)) \sum_{N_{1}} \mathbf{P}\left\{\lambda^{(n, m)}=N\right\}=1+o(1),
$$

а для суммы $S_{2}$ справедлива оценка (33). Отсюда вытекает утверждение теоремы 3.

\section{Список литературы}

1. Павлов Ю. Л., Предельные теоремы для числа деревьев заданного объема в случайном лесе. Матем. сб. (1977) 103, №3, 392-403.

2. Павлов Ю. Л., Асимптотические распределения максимального объема дерева в случайном лесе. Теория вероятностей и ее применения (1977) 22, №3, 523-533.

3. Павлов Ю. Л., Случайıые леса. Петрозаводск, 1996.

4. Грэхем Р., Кнут Д., Паташник О., Конкретиая математика. Мир, Москва, 1998.

5. Чеплюкова И. А., Один случай предельного распределения числа циклических вершин в случайном отображении. Дискретиая математика (2004) 16, №3, 76-84.

6. Колчин В. Ф., Случайıые графы. Физматлит, Москва, 2000.

7. Сачков В. И., Вероятиостиые методы в комбипаториом апализе. Наука, Москва, 1978.

Статья поступила 14.03.2005. 